\title{
On teaching articulatory phonetics via an orthography
}

\author{
BRIAN BYRNE \\ University of New England, Armidale, New South Wales, Australia
}

\begin{abstract}
In six experiments, the ability of university students to obtain generalizable knowledge about subphonemic distinctive features through learning to read an orthography based on these features was explored. In the reference experiment, it was found that learning four grapheme-phoneme pairs did not generate "feature awareness," as tested by generalization to new instances of the orthography. In a second experiment, it was demonstrated that this failure was not due to inherent limitations in the testing technique, and a third experiment indicated that a considerable increase in exposure to the orthography and its principles did not help. In the last three experiments, instruction in articulatory phonetics was given prior to subjects' learning the orthography, but reliable generalization to new instances emerged only when the instruction was based directly on the phonemes used in acquisition. Also, successful generalization was found to go hand in hand with verbalizable awareness of phonetic structure. A high level of performance was achieved only when the orthography/speech isomorphism was pointed out clearly. The results indicate that usable knowledge about an implicitly known property of speech is relatively resistant to instruction via an orthography, and that direct instruction in the property is necessary. An analogy is drawn with the acquisition of reading by nonreaders, with the results of the study lending weight to the use of curricula that emphasize phonemic awareness.
\end{abstract}

There is considerable interest in the question of how people may "access," or gain knowledge about, the elements and rules of language, normally not the subject of conscious contemplation and normally not recruited for purposes outside the expressive and communicative functions of language itself (Byrne, 1981; Rozin, 1975). In this paper, I report a series of experiments in which attempts were made to develop in subjects generalizable knowledge about one aspect of linguistic organization, the voicing contrast in articulation. The vehicle for the attempted instruction was an orthography that embodied this and other aspects of the subphonemic feature matrix.

Interest in knowledge about the sounds of language follows naturally from analyses of reading acquisition. There is now considerable evidence that learning to read carries with it a heightening of "phonemic awareness," the realization that there are stable articulatory/acoustic units below the level of the word, namely, phonemes. A study by Morais, Cary, Alegria, and Bertelson (1979) is a particularly good example of this phenomenon. In that research, it was shown that previously illiterate adults taught to read were better able to segment words on phonemic prin-

I thank Douglas Tegart for preparation of the figures. I am also grateful to $\mathrm{L}$. Brooks and an anonymous reviewer for insightful comments on an earlier version of this paper, and to D. Nixon and C. Edmonds for telling me about shorthand systems. My mailing address is: Department of Psychology, University of New England, Armidale, New South Wales 2351, Australia. ciples than were a matched group of illiterates. Byrne and Ledez (1983) obtained confirmatory evidence. Also, Williams (1980) demonstrated that children with reading difficulties could be assisted by exercises that enhanced phonemic awareness, and a final piece of evidence that suggests that knowledge of orthographic and phonemic structure go hand in hand comes from Ehri and Wilce (1980), who showed that knowing how to spell a word influences a subject's conception of its phonemic shape-fourth-graders, for example, were aware of the alveolar tap in "pitch" but not in "rich." It does appear that even very young children, prior to reading instruction, are sensitive to some surface properties of words (e.g., rhyme-Lenel \& Cantor, 1981), and, in the research of Byrne and Ledez (1983), very weak adult readers showed awareness of the major break in words between initial consonation and vowel group, although more fine-grained discriminations were lacking. But with learning to read seems to come a filling out of the details of sound structure, down to the level of the phoneme. It appears that an alphabetic orthography is a convenient device for developing insights into an aspect of language partially hidden prior to reading instruction.

How easy is it to teach people aspects of phonology via an orthography? Evidence from research into reading failure suggests that for some children, at least, it is quite difficult. Work by Snowling (1980) suggests that developmental dyslexics do not fully understand the internal structure of either spoken or written words-what reading skill they have rests 
upon unanalyzed links between print and speech at the level of the word. Point-by-point contact between orthography and speech at the lowest available level (grapheme and phoneme) is not well established in these children. Bradley and Bryant (1978) also found that, for children behind their age norms in reading, a fair level of reading skill can be in evidence in the absence of a good grasp of "auditory organization." For example, the subjects had difficulty in telling that the odd word in "weed," "peel," "need," "deed" is "peel," with the words presented auditorily. Identifying the personal, social, and educational factors contributing to this dissociation of reading skill and linguistic insight is a major research task, of course, but it may help to have laboratory investigations of factors controlling the achievement of insight. The following studies represent a start on that program, using college students as subjects.

Because literate adults already know about phonemic segmentation, it was decided to try to teach some aspects of articulatory phonetics at the level of distinctive features. By doing so, it was hoped to supplement data from existing experiments in which adults learn a new writing system. Brooks (1977) reported an extensive series of studies using artifical alphabets. He showed, among other things, that although having a functional, but unfamiliar, alphabet assists subjects in learning to identify words fluently (as against acquiring similar word-symbol associations not mediated by the alphabetic principle), various manipulations of the visual symbols, which provided sound-correlated visual patterns, also helps at achieving fluency. This opens up the intriguing possibility that the same might be true of initial or remedial reading instruction, namely, that learners could be assisted by sound-correlated visual patterning. But as Brooks pointed out, his subjects had already used an alphabet, whereas children have not, and the effects of visual pattern may be quite different in subjects for whom "linguistic translation" (the alphabetic principle) is not yet a reality. In Brooks's experiments, the translation procedure was either present or not in the stimulus-response pairs, and if present, it was explicitly shown to the subjects. In the current studies, the symbols and sounds were always linked systematically, and the question was whether, and under what circumstances, subjects could in some sense discover the translation procedure. Although in my experiments visual pattern was not selected as a variable of interest, once the experimental paradigm has been established, future studies can be directed at investigating pattern, and other things, in a situation one step closer to that of the initial reader, namely, the acquisition of an orthography without prior experience with the sound structures involved, except as implicit aspects of language.

An orthography that represented a portion of the feature matrix was designed. Each phoneme was rep- resented by a two-part symbol, one component signifying place of articulation, the other, voicing. Like the alphabet, the orthography was arbitrary but consistent. The system is illustrated for four phonemic segments in Figure 1. The two alveolar fricatives ( $z$ and s) share the same symbol in the upper position, as do the two bilabial stops (b and p). Likewise, the voiced sounds ( $z$ and $b$ ) share the lower component, while the unvoiced segments also have a common element in that position. It needs to be recognized that the orthographic change in the upper position actually correlates with more than a change in place of articulation. It also corresponds to changes in the following features: continuant, strident, and coronal. Hence, "place" is used in a shorthand manner in this paper. However, because orthographic variation also was pointed out as corresponding to place variation in some of the later experiments utilizing explicit instructions to subjects, place is a reasonably suitable code word in this series. In all experiments except Experiment 2, a control condition, subjects were taught the orthographic system to certain criteria and then tested for generalization to partially new symbols. Each of these generalization stimuli had either the voicing or nonvoicing component, plus a new upper element, and subjects were forced to choose between a voiced and unvoiced segment corresponding to each symbol. For example, subjects were told that a new symbol represented either $/ v /$ or $/ f /$. The correct response was $/ v /$ if the stimulus contained the voicing element and /f/ if it had the component for nonvoicing. Subjects for whom the acquisition of this orthographic fragment was based upon the most analytic mapping possible, that is, point-to-point contact between components of the writing system and distinctive features, would have a basis for choosing correctly. Nonanalytic correspondences established at a one-to-one level (entire "grapheme," entire phoneme) would be useless as bases of choice, and subjects would be forced to guess.

The choice of the voicing contrast was dictated by two considerations. First, it is known that the presence or absence of voicing is highly salient, perceptually (Miller \& Nicely, 1955). Perceptual confusions tend to be made within, but not across, the categories of [+ voice] and [-voice]. Language users have a good implicit grasp of the contrast. Second, and on a more practical level, there are eight pairs of phonemes differentiated solely in terms of voicing used in English, yielding enough stimuli for the requirements of the study - a training phase and a generalization phase. Choosing certain other distinctive features would be very restrictive. Imagine, for example, attempting to train and test for knowledge of the bilabial feature. At least two examples are required for training in what is essentially a concept-learning experiment (e.g., /b/ and /p/), leaving only one phoneme $(/ \mathrm{m} /)$ as the test stimulus. Naturally, the choice of just one aspect of articulatory phonetics as the 
basis for testing restricts the generality of any conclusions from the results. What is discovered to be true for voicing, in terms of the ease or difficulty of subjects' successfully generalizing to new items, may not be the case for other phonetic constituents, such as stridency and nasality. Nevertheless, for the reasons outlined above, it was considered that voicing was a useful starting point for this research.

The first study served as a reference experiment for the whole series. In it, subjects were taught the orthography of Figure 1, and then tested on the pairs $/ \mathrm{v}-\mathrm{f} /$, $/ \partial-\Theta /, / g-k /$, and $/ d-t /$. Judging from the chancelevel performance on the test phase and from subjects' comments on their performances, there was no evidence of generalizable knowledge of orthographic structure. The remaining experiments, then, were $\mathrm{d}_{\mathrm{i}}$ rected mostly at determining what training conditions, if any, would result in the development of this knowledge.

If subjects were found to be able to successfully generalize to new orthographic symbols, the question of how best to characterize the transfer would still be open. One traditional concern is whether the knowledge is conscious or unconscious, that is, explicit or implicit. Recently, Brooks (1978) argued for a further distinction, between rule-based and instance-based judgments. In the former, subjects are operating on the basis of an analytic procedure by which aspects of a stimulus are recognized as predicting category membership, as in the usual laboratory concept-learning studies. In the instance-based process, a new item is assigned a category on the basis of its similarity to a known individual, an operation that does not require the analysis of items into criterial attributes. In order to provide preliminary information about the nature of any successful transfer, subjects throughout these experiments were presented with open-ended questions to elicit subjective reports about how they performed the tasks.

\section{EXPERIMENT 1}

\section{Method}

Subjects. Eight undergraduate and graduate students in psychology served on a volunteer basis. None had had any training in phonetics or psycholinguistics.

Materials. The four symbols in Figure 1a were printed on separate $12 \times 7.5 \mathrm{~cm}$ cards. Also on individual cards were the 16 possible consonant-vowel-consonant (CVC) syllables generated from the four consonants plus the vowel /a/. Samples are in Figure 1b. For the test phase, the syllables shown in Figure 2 were created, each containing one new symbol.

Procedure. The subjects were tested individually. The four cards containing the symbols for the four consonants were laid out in a row, after the experimenter (author) had described the general purpose of the experiment (to teach part of a new writing system). The experimenter made each sound in turn. Then the cards were randomized and laid down one at a time, the experimenter again producing the appropriate phoneme (in the case of $/ b /$ and $/ p /$, along with the schwa vowel). The cards were again shuffled, and the subject was asked to produce the consonant for each symbol

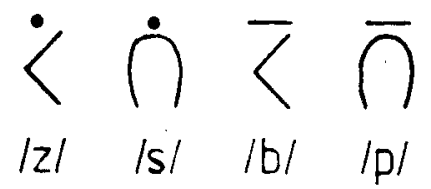

(a)
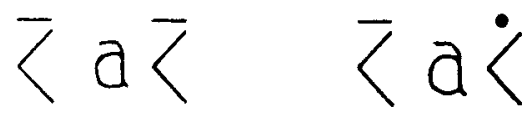

lbabl

/bæzl
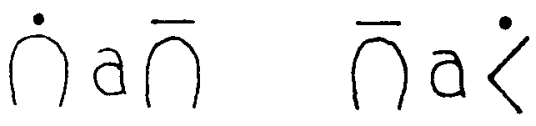

/s ap/

|p æez/

(b)

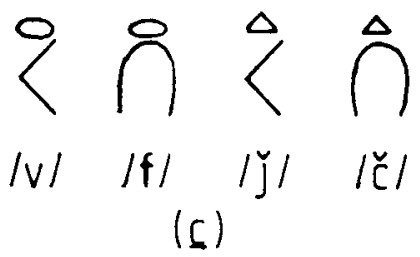

Figure 1. Symbols and corresponding phonemes and syllables used in experiments. (a) Training phonemes, Experiments 1, 3, 4, 5, and 6. (b) Examples of training syllables, Experiments 1, 3, 4, 5, and 6. (c) Additional phonemes, Experiment 3.
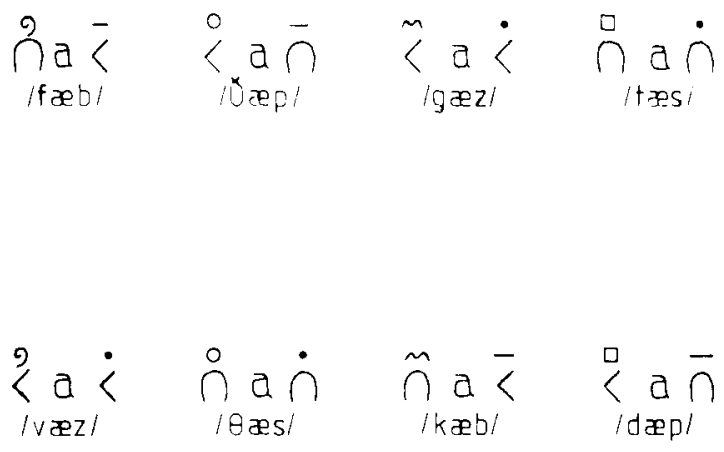

Figure 2. Syllables used in generalization (test) phase, Experiments $1,3,4,5$, and 6 .

as they were laid in a stack. Corrective feedback was provided as necessary. This aspect of the training was continued until the subject managed three successful, successive passes through the fouritem list.

Training continued with the 16 syllables. The first of the randomized series was read to the subject, who then was required to decode the remainder. Again, the subject's responses were corrected, if needed, and the criterion was one error-free pass through the entire stack. The cards were shuffled between list presentations. 
In the test phase, the subject was instructed as follows: "I will now show you some new syllables, each containing one new symbol. I will give you two alternative possibilities for the syllables, and I want you to try to work out which of the two the new syllable is. If you can't work it out, have a guess."

Each generallzation syllable was presented in turn, in the same order as in Figure 2, across the rows. With each one, the experimenter said, "This is either /fab/ or /vab/ (as appropriate), /freb/ or /vab/. Which do you think?" The subject's response was recorded; no feedback was given.

After being tested, each subject was interrogated, first about any system he or she had used in the generalization phase, and then about whether he or she had detected any system in the original orthography.

\section{Results and Discussion}

The mean number of correct responses at generalization was 4, exactly chance, with a range of 3 to 6 and a standard deviation of 1.07 . Not one subject gave any indication of understanding the systematic relationship between the orthography and the feature structure of the phonemes. Most of the subjects claimed that they sought visual clues from the new (and irrelevant) component of the symbol. For example, one subject claimed that the new upper component for /f/ and /v/ "looked more like" /f/. Many noted that the same new symbol reappeared later in the test phase, and that they had switched their choice to the other sound if they could remember the first choice. For two subjects, there seemed to be some glimmerings that sound relationships provided a sensible basis for choice. One, for instance, said that the unvoiced symbol seemed to "go with soft sounds," which permitted her to select correctly /f/ versus / $/$. But this weak insight was not carried through to other items-her score was 3 out of 8 in generalization.

The subjects varied considerably in how quickly they learned the associations and their use in syllables. The number of errors made by subjects in reaching criterion in the two stages of acquisition, phoneme and syllable responses, was selected as the primary measure of learning. It is more sensitive than trials to criterion-the same number of trials can contain considerable variation in individual errorsand the highly significant correlation of $.603(p<.001)$ between errors in the two stages, over all subjects tested in the series, justifies summing error types. The number of errors in Experiment 1 varied from 0 to 55 (mean =15.1). Given the relatively homogeneous nature of the subjects and the small number (four) of stimulus-response links to be acquired, both the range and number of errors seem surprising. There may quite different learning strategies at work across the subjects, a topic that will be raised again. By the time each subject reached criterion, however, he or she was quite fluent at "reading" the syllables.

For the sake of completeness, the median trials to criterion in the learning phase of the acquisition process (basic associations) for all experiments are presented in Table 1. Medians were chosen because of
Table 1

Median Trials to Criterion in Leaming Basic Associations (Minimum = 3)

Experiment

3

\begin{tabular}{ccccccc}
1 & 2 & 1 st Set & 2nd Set & 4 & 5 & 6 \\
\hline 9 & 10 & 7 & 8.8 & 6.5 & 7.1 & 7.5 \\
\hline
\end{tabular}

the high fluctuations across subjects in trials to criterion, and primary acquisition was selected simply because it is the usual measure in studies of this sort. The comments on learning rates made throughout the paper, which are based on total errors in both stages, are in general confirmed by the data of Table 1 . Average learning time seems relatively unaffected by variations in material and instructions.

In sum, it appears that relatively articulate and thoughtful subjects can learn symbol-sound associations that are mediated by a principled orthography without discovering the orthographic principle, as indicated by their failure to decode new instances containing one aspect of the code.

\section{EXPERIMENT 2}

It is easy to think of a range of reasons why subjects failed to generalize to partially new symbols in Experiment 1. Apart from the theoretically interesting possibility that implicit knowledge of phonetic structure may not be made usable by the acquisition of an orthography, it may be that there are features of the particular training and generalization technique chosen that inhibit the emergence of learning. For instance, the number of stimuli and responses may be too small to require systematic analysis by subjects-knowledge of rules of correspondence at the lowest level may help only when associations exceed some critical number, larger than four. If it could be shown, however, that generalizable learning does take place with these stimuli when the responses are transparent in terms of constituent structure, then the argument that failure of generalization in Experiment 1 was due to an insensitive technique would be undermined. The aim of Experiment 2 was to examine this possibility.

\section{Method}

Subjects. Eight volunteers, selected on the same basis as those in Experiment 1, were used.

Materials. Precisely the same basic stimuli were used. This time, however, they represented the words "fan," "fit," "pan," "pit" instead of the phonemes $/ z, s, b, p /$. For literate subjects, the division of words into initial consonant and vowel groups should be quite straightforward, and, hence, if they were motivated to discover analytic correspondences between the orthography and the response words, they should be able to do so readily. In place of syllable decoding, the 16 possible two-symbol combinations were presented as phrases: two symbols separated by an 
ampersand, and read as "fan and pit," and so on. The eight generalization stimuli were constructed analogously to those in Experiment 1-a two-part symbol, the upper of which was new, the lower one representing either the "an" or the "it" vowel group.

Procedure. The basic procedure was the same as that in the first study-a two-stage acquisition period (isolated words, then phrases), to the same criteria. Generalization was preceded by the same instructions, with the following words being provided as alternatives from which to choose upon presentation of each partially new symbol (correct answer in italics): man mit; ban bit; tan tit; nan nit; lit lan; dit dan; git gan; rit ran.

\section{Results and Discussion}

Mean number of correct generalization responses was $6.75(85 \%), \mathrm{SD}=1.49$, and a $t$ test comparing the mean with chance $(4)$ yielded $t(7)=5.227(p<.01)$. All but two subjects indicated a clear conception of the system linking symbol and word. They clearly recognized the components of the words and how these components were linked to the parts of the symbols. Four subjects scored perfectly, three managed 6 correct, and one, 4 . This latter subject and one of those who scored 6 were the two who gave no indication of understanding the orthography's basis. Mean number of errors during acquisition was 33.6 (range $=6$ to 78 ).

It seems that when a systematic basis for acquisition of a four-item orthography is available and obvious, subjects on the whole will use it. The facts that two apparently failed to see the system and that two who did see it failed to score perfectly point, in turn, to interesting individual differences in learning strategies and to the possibility that temporary attentional and memory factors may mar performance for some subjects. But it is reasonable to suppose that the generalization failure in Experiment 1 was not an uninteresting product of the training and testing technique used. At this stage, it seems more likely to have been due to difficulty in becoming aware of the feature network underlying phonemes or in detecting the appropriate contrast in new phoneme pairs.

\section{EXPERIMENT 3}

In this study, two things were changed from the reference experiment. One was the number of phonemes included in the training phase-increased from four to eight. The other was the criterion that subjects were required to reach-three successive, successful passes through each of two syllable lists. In the course of the experiment, it became clear that this criterion was simply too demanding for most subjects, and it was relaxed slightly to permit one error per pass. However, the net effects of the changes were to lift quite considerably the demands on the subjects and to give them greater experience with the orthography prior to generalization. The question being addressed was: Given the apparent difficulty in understanding the orthography evident from the reference experiment, would an increase in exposure to it, via a larger number of exemplars and more sustained use, lead to analytic learning? I admit that in science it is desirable to change things singly, and a conservative approach in this series would have been to increase the number of items or change the acquisition criterion, but not both. But one can always backtrack and run the necessary isolating experiments if significant generalization does occur. If it does not, then time and subjects have been saved.

\section{Method}

Subjects. A new group of eight volunteer undergraduate and graduate students served as subjects.

Materials. The single and syllabic stimuli from Experiment 1 were used, along with four new symbols, representing the sounds $/ v, f ; j$, č/ (see Figure 1c). The 16 consonant-vowel-consonant (CVC) syllables derivable from these sounds [plus /a/] were printed on cards. Generalization stimuli were similar to those in Experiment 1, except that the pair /v, f/ was replaced by $/ \check{z}, \check{s} /$, since the former were training stimuli.

Procedure. The subjects first learned the four symbols from Experiment 1, except that the criterion at syllable decoding was three successive, successful passes through the stack (one error per pass permitted). After a short rest, the subjects went through the same acquisition stages for the four new symbols. Generalization was tested with the standard instructions, and the usual interrogation followed the completion of testing.

\section{Results and Discussion}

Of central interest is the mean number of correct generalization responses, which was $4.6(58 \%), \mathrm{SD}=$ $1.59[t(7)=1.10, p>.30]$. One subject, who scored 7 at generalization, clearly understood the orthography's basis. He reported that the voiced symbol represented "harsh" sounds and the nonvoiced symbol, "soft" sounds. The remainder gave subjective reports very much along the lines of those in Experiment 1 , that is, with attention directed to possible visual clues in the transfer items. Thus, the interesting possibility of individual differences again is raised. On the whole, however, it appears that even the relatively high demands made on the subjects in this experiment, conditions under which deciphering the systematic links between symbol and sound would presumably cut the memory load considerably, failed to induce generalizable acquisition.

The error patterns of subjects were similar to those in Experiment 1. They made an average of 13.3 wrong responses in reaching criterion on the first four sounds and 11.1 on the second group of sounds.

\section{EXPERIMENT 4}

This experiment and the next two had a common feature: Prior to learning the orthography, the subjects were presented with a minilecture on articulatory phonetics. One of the commonsense claims 
within the literature on reading instruction is that the skill should be taught as directly and explicitly as possible, and this includes making the child aware of speech segmentation at the appropriate (phonemic) level (Rozin \& Gleitman, 1977). So far, in the experiments in this series, subjects had not been able to understand the internal organization of the orthography as it corresponded to components of sound, despite successfully having learned to use the orthography. They were in a position analogous to that of children who learn that CAT says "cat" and BAT says "bat" but who have not mastered graphemephoneme correspondences. One would assume that these children would exhibit their ignorance by not being able to tell whether FAT says "fat" or "fin," for example, if they had not specifically learned FAT and were forced to guess. So it may be that instruction in subphonemic features provides a suitable base upon which to build analytic understanding, just as in the case of reading-disabled children (Williams, 1980). In this experiment, the subjects were instructed in pertinent aspects of voicing and place of articulation, illustrated by phonemes not later incorporated into either training or generalization phases. Is it sufficient to alert subjects to the distinctive feature system in this indirect way to enable them to acquire baselevel correspondences between symbols and sound?

\section{Method \\ Subjects. In this study, 10 volunteers were recruited. \\ Materials. Training stimuli were those of Experiment 1. Gener- alization stimuli were slightly different because the contrasting pair of $/ \mathrm{v} /$ and $/ \mathrm{f} /$ were part of the instruction in phonetic struc- ture.}

Procedure. The subjects were presented with the following lecture prior to training and generalization; otherwise, the procedure was identical to that in Experiment 1:

\footnotetext{
"Before we start the experiment, I want to draw your attention to some of the properties of the sounds of language. You may wish to keep them in mind during the experiment.

"The sounds of our language are built up from basic components, to which linguists give the name 'distinctive features.' For example, /f/ and / $/$ are both made by placing the lower lip against the upper teeth and expiring air. They have the same 'place of articulation,' as it is called [subject checks]. But they are different in one way. If you put your hand on your voice box and say $/ v \ldots /$, you can feel vibrations. But say $/ \mathrm{f} . . . /$, and there's no vibration [experimenter demonstrates, and subject checks]. In the case of $/ v /$, the vocal cords are drawn across the windpipe, and made to vibrate. This gives the sound a 'voiced' quality, and that's what linguists called ita voiced sound. For $/ f /$, the vocal cords are relaxed, and don't vibrate-air passes through them without affecting them. /f/ is 'unvoiced.' So / $/$ and / $f /$ are the same in terms of 'place of articulation' (teeth/lip combination) and different in 'voicing.'

"Another example is $/ \check{C} /$ and $/ \dot{j} /$. They're made by forcing air between tongue and hard palate, just behind the teeth. They're known as 'palatals' [subject checks]. But they too differ in voicing $-/ j /$ is voiced, $/ \check{c} /$ is unvoiced [subject checks]. So, same in place of articulation (palate), different in voicing. All sounds can be classified in terms of features like voicing, place of articulation, and a few other things."
}

\section{Results}

On the average, there was no evidence of successful generalization: The mean number of correct responses was $4.3(54 \%), \mathrm{SD}=1.16[\mathrm{t}(9)=0.82, \mathrm{p}>.40]$. One subject, who scored 7 , did demonstrate comprehension during interrogation. Some of the other subjects focused on visual form (as in the reference experiment), although some indicated glimmerings of understanding. One, for instance, realized that the voicing symbol should have represented voicing because $/ z /$ is the voiced member of the $/ z, s /$ pair. However, she failed to realize that the same relationship held between $/ b /$ and $/ \mathrm{p} /$. This suggests that some contrasting pairs may be better vehicles for inducing realization of subphonemic feature structure than others, and invites further research. But, it appears that drawing subjects' attention to the voicing contrast in a general sense is not sufficient for them to understand how it is embodied in an orthography.

The mean number of errors prior to reaching criterion was 9.6, with the usual big range (0-31).

\section{EXPERIMENT 5}

The instruction in voicing and place of articulation in this study used $/ z, s ; b, p /$ as the examples and as the phonemes in the training phase. Except for this change, the lecture was identical to that in Experiment 4 . The aim, of course, was to teach the feature system quite directly without actually pointing out the links between writing system and sound.

\section{Method}

Subjects. On this occasion 12 volunteers were used.

Materials and Procedure. These were the same as those used in Experiment 4, except for the substitution of $/ z, s ; b, p /$ in place of $/ v, f ; j, \grave{c} /$ in the minilecture on phonetic structure.

\section{Results}

Mean generalization score was $5.4(68 \%)$, which was significantly above chance $[t(11)=3.02, p<.05]$. Five of the subjects gave no indication of having seen symbol/feature correspondences, and the mean score of these five was 4.0. The remaining seven all revealed some understanding of the system, although not all could carry through their insights to all the generalization stimuli. The mean score of this group was $6.4(80 \%)$. Clearly, a worthwhile research project would be to look for characteristics of subjects who transfer successfully and those who do not. Perhaps Baron's (e.g., Baron \& Strawson, 1976) work, in which analytic and holistic styles of treating print have been isolated and examined, may provide a starting point for such a project.

It does seem clear from this and the earlier experiments that generalizable knowledge and explicit awareness go together. Only subjects who could give some verbal account of the orthographic principle 
showed generalization. There seems to be no "learning without awareness" for these subjects and this material. Furthermore, the reports that successful subjects gave were reminiscent of analytic, rule-based learning, not instance-based learning. That is, the subjects generally recognized that one member of each transfer pair was voiced and the other was not and knew which symbol predicted voicing and which predicted nonvoicing. They did not say things like "it must be $/ \mathrm{v} /$ because it sounds like $/ z /$." This is hardly surprising, given that the component structure of the responses was emphasized in the minilecture.

Whether nonanalytic transfer will show up under other experimental circumstances using this kind of material remains to be seen.

The fact that about half of the subjects acquired systematic orthography/sound links did not result in faster learning. The mean number of errors prior to criterion was 16.7 (15.1 in Experiment 1), and there was no systematic relationship between errors during training and subsequent score in generalization $(\mathrm{r}=.27)$.

When instruction in phonemic structure is made in the context of the actual segments to be used in training, it appears that significant generalizable knowledge develops, although the learning process itself is not speeded up.

\section{EXPERIMENT 6}

There seem to be two reasons why the group average in Experiment 5 was not higher than it was. One is that some subjects failed to discover the translation procedure, and the other is that the seven subjects who did succeed may have found difficulty in deciding which of a new minimally contrasting pair of phonemes was voiced. In Experiment 6, an attempt was made to ensure that all subjects were clear about orthographic and phonemic structure and their relationship by (1) providing the minilecture, as in Experiment 5 , and (2) telling subjects directly what each component in the orthography represented. It was likely to be the case, therefore, that failures at generalization would be attributable to discrimination problems with transfer stimuli (or perhaps attentional or memory deficits operating during the test phase) rather than to subjects' not understanding the orthography.

\footnotetext{
Method

The eight subjects in this condition followed the procedure of Experiment 5. In addition, they were told what each component of the orthography represented, in sentences such as "This symbol [experimenter pointing to voicing sign] stands for a voiced soundit appears whenever the sound is voiced." Similar instructions for place were given. The subjects were rerninded of the system's properties at several stages during training. As usual, the generalization test was conducted without feedback.
}

\section{Results and Discussion}

The subjects achieved a high level of performance of $7.5(94 \%)$ correct responses in generalization $[t(7)=$ $13.09, \mathrm{p}<.001]$. The average score in this experiment exceeded the mean in Experiment $5(5.4)[t(18)=3.86$, $\mathrm{p}<.01]$. It seems that the voicing contrast can be detected in new consonant pairs and linked to the orthography, although some errors remain (three subjects scored less than perfectly). Whether these difficulties are perceptual, attentional, or memorial in nature is not known.

Interestingly, learning was no quicker for these subjects than for those in previous groups. The mean number of errors prior to criterion was 14.3 (15.1 in the reference experiment), with a range of 2 to 27 . Although the course of acquisition needs much more study, especially in view of the vast differences among subjects in learning rate, there is no obvious advantage conferred by knowing the orthography's systematic base, at least for the training conditions established for these experiments.

\section{GENERAL DISCUSSION}

The overriding impression from the experimental series is that it was quite difficult for subjects to notice the systematic basis of the orthography. This did not seem to be attributable to an inherent insensitivity of the techniques of training and generalization (Experiment 2). Rather, it probably was due to difficulty in becoming explicitly aware of subphonemic feature structure, or at least of the voicing contrast. With these subjects and these conditions of acquisition, it was not until attention was drawn to the constituents of the phonemes used in acquisition that reliable generalization emerged (Experiment 5), and only when the details of the orthography/sound mapping were made quite explicit did a high level of performance appear (Experiment 6). Neither increasing the size of the learning set and exposure to the system (Experiment 3) nor indirect instruction in phonetics (Experiment 4) helped.

The generality of these conclusions cannot be assumed. Other feature categories (e.g., nasality, stridency) may be more transparent to subjects. Other training techniques (exposure, arrangement of item presentation in systematic ways, orthography design, etc.) may produce different results. And, of course, subjects with different backgrounds may perform in different ways, since evidence of individual differences already exists in these data. Nevertheless, we do know that students of linguistics and psycholinguistics can readily enough learn about voicing when told directly. It seems that this orthography, unsupported by direct instruction, is not a suitable vehicle for successful instruction.

On the question of visual pattern, it is interesting 
to note that Pitman shorthand represents voicing systematically, and that the device for doing so is pairs of otherwise identical heavy and light strokes for voiced and unvoiced phonemes, respectively. In the Gregg system, line length carries voicing information, the longer line being the voiced sound. One might suspect that these choices of correspondence were not purely coincidental, that there is something "appropriate" about a dense or longer symbol's representing voicing. Also, the Korean alphabet is based partly on symbols that are meant to mimic articulatory positions assumed when making the sounds in question (Taylor, 1981). The fact that in Brooks's (1977) experimental series visual pattern was shown to affect fluency makes plausible the supposition that it may also affect the discovery of symbol-sound correspondence, and, speculating on the bases of shorthand and Korean writing, one factor guiding the search for an optimal orthography may be "appropriateness."

Even if it were the case that a change in experimental conditions resulted in successful generalization, this would leave one important conclusion from the present series intact: When "feature awareness" cannot be induced simply by orthographic training, instruction in the appropriate aspects of the sound system can help. Imagine, for example, that subjects could successfully choose which of two new sounds, one a fricative and the other not a fricative, was represented by a partially new symbol after the subjects had acquired an orthography that embodied that aspect of manner. Even so, the fact that not all aspects of phonetic-feature structure can be made transparent by orthographic training, including the basic distinction of \pm voice, would remain interesting, and what further conditions of training may be necessary to generate analytic understanding is a sensible question to ask and explore. It is made the more compelling by evidence, reviewed earlier, that some children develop a nonanalytic reading style, which hampers them in deciphering new instances (words). If we wish to mimic in the laboratory such reading styles, we need to find phonetic features that cannot easily be brought to awareness by the learning of an orthography based on them. In that sense, it is rather similar to determining exposure durations in tachistoscopic experiments that result in moderate error rates to assess the influence of variables other than duration. The fact that certain exposure times result in errorless performance and others in nothing but error may have its own inherent interest, but is ignored in certain research contexts. Similarly, students of speech perception may be interested to know which features are easy to learn about and which are difficult, but someone trying to build a laboratory analogue to beginning reading will be content with lo- cating a feature that behaves as voicing did in these experiments.

The foregoing discussion raises the question: Are there any lessons for the teaching of reading? Caution is in order here. Any claims about the principles underlying reading acquisition have to be tested, in the long run, with the subjects and materials actually involved in the process. There are too many indirectly plausible techniques littering the history of reading instruction. Moreover, the perceptual transparency of the feature, any feature, may be orders of magnitude below that of the phoneme, and it is this opacity that stands out in the current research. But, insofar as analogues are useful, it can be said that these data support the view that teaching children about phonemic segmentation is an important adjunct to reading instruction (Rozin \& Gleitman, 1977; Williams, 1980). This might be especially so in those cases in which reading retardation is in evidence, which are also often the cases in which explicit understanding of the phonemic principle is most lacking (Bradley \& Bryant, 1978; Byrne \& Shea, 1979; Fox \& Routh, 1980).

The results also serve as a reminder that using an orthography does not require an understanding of its most basic isomorphism with speech. The nongeneralizing subjects can be said to have used the system in the same way as a pure "whole-word" reader can be said to "read." Given what has been demonstrated about the learning strategies of these test-sophisticated subjects, perhaps it is not surprising that some children do not detect the appropriate mapping between print and speech and choose, instead, to build links between them at the salient level of the word. Snowling's (1980) research with developmental dyslexics demonstrated that that level forms the basis of their reading, not direct graphemephoneme correspondences. One might speculate that initial success attainable with high-level correspondences (whole-word reading) when a small number of words are involved could seduce learners into adopting that strategy in favor of a more analytic approach, which yields the correct pronunciation of printed matter only with considerably more effort early in acquisition. The remarks of one subject from Experiment 1 are revealing. She needed a very large number of trials to reach criterion during training (she made 55 errors). She claimed that she suspected there must be a system embodied in the orthography and expended a great deal of effort in trying to discover it. She was eventually unsuccessful (generalization score, 3 of 8). In hindsight, she felt she would have been better off learning the associations "off by heart." Analytic acquisition imposes a burden on the learner in cases in which the constituents of (at least) one side of the association are not readily perceived, even 
though it is in the long run the efficient strategy. We should be aware of this in teaching reading.

\section{REFERENCES}

Baron, J., \& Strawson, C. (1976). Use of orthographic and wordspecific knowledge in reading words aloud. Journal of Experimental Psychology: Human Perception and Performance, 2, 386-393.

Bradley, L., \& Bryant, P. E. (1978). Difficulties in auditory organization as a possible cause of reading backwardness. Nature, 271, 746-747.

Brooxs, L. (1977). Visual pattern in fluent word identification. In A. S. Reber \& D. L. Scarborough (Eds.), Toward a pyschology of reading: The proceedings of the C.U.N.Y. conferences. Hillsdale, N.J: Erlbaum.

Brooks, L. (1978). Nonanalytic concept formation and memory for instances. In E. Rosch \& B. B Lloyd (Eds.), Cognition and categorization. Hillsdale, N.J: Erlbaum.

BYrNe, B. (1981). Reading disability, linguistic access, and shortterm memory: Comments prompted by Jorm's review of developmental dyslexia. Australian Journal of Psychology, 33, 83-95.

Byrne, B., \& Ledez, J. (1983). Phonological awareness in readingdisabled adults. Australian Journal of Psychology, 35, 185-197.

Byrne, B., \& SheA, P. (1979). Semantic and phonetic memory codes in beginning readers. Memory \& Cognition, 7, 333-338.

EняI, L. C., \& WILCE, L. S. (1980). The influence of orthography on readers' conceptualization of the phonemic structure of words. Applied Psycholinguistics, 1, 371-385.

Fox, B., \& Routh, D. K. (1980). Phonemic analysis and severe reading disability in children. Journal of Psycholinguistic Research, 9, 115-119.
Lenel, J. C., \& CANTOR, J. H. (1981). Rhyme recognition and phonemic perception in young children. Journal of Psycholinguistic Reseach, 10, 57-68.

Miller, G. A., \& Nicely, P. (1955). An analysis of perceptual confusions among some English consonants. Journal of the Acoustical Society of America, 27, 338-352.

Morais, J., Cary, L., Alegria, J., \& Bertelson, P. (1979). Does awareness of speech as a sequence of phones arise spontaneously? Cognition, 7, 323-331.

Rozin, P. (1975). The evolution of intelligence and access to the cognitive uncounscious. In J. M. Sprague \& A. N. Epstein (Eds.), Progress in psychobiology and physiological psychology (Vol. 6). New York: Academic Press.

Rozin, P., \& Gleitman, L. R. (1977). The structure and acquisition of reading II: The reading process and the acquisition of the alphabetic principle. In A. S. Reber \& D. L. Scarborough (Eds.), Toward a psychology of reading: The proceedings of the C.U.N.Y. conferences. Hillsdale, N.J: Erlbaum.

Snowling, M. J. (1980). The development of grapheme-phoneme correspondence in normal and dyslexic readers. Journal of Experimental Child Psychology, 29, 294-305.

TAYLOR, I. (1981). Writing systems and reading. In G. E. Mackinnon \& T. G. Waller (Eds.), Reading research: Advances in theory and practice (Vol. 2). New York: Academic Press.

Williams, J. (1980). Teaching decoding with an emphasis on phoneme analysis and blending. Journal of Educational Psychology, 72, 1-15.

(Manuscript received March 10, 1983; revision accepted for publication October 24, 1983.) 